\title{
Análise da repentina duplicação da largura de um poço quadrado infinito 1-dim: um resultado surpreendente à primeira vista
}

Analysis of a sudden doubling of the space between walls of an infinite 1-dim square well: a surprising result at first

\author{
Rogério Netto Suave ${ }^{1}$, Erich Andrade Ferreira ${ }^{1}, J_{0 s e ́}$ Alexandre Nogueira* ${ }^{*}$ \\ ${ }^{1}$ Universidade Federal do Espírito Santo, Departamento de Física, Centro de Ciências Exatas, Vitória, ES, Brasil.
}

Recebido em 17 de outubro de 2019. Revisado em 04 de dezembro de 2019. Aceito em 17 de dezembro de 2019.

\begin{abstract}
Neste trabalho, chamamos a atenção de como um exercício comumente sugerido em livros-texto de Mecânica Quântica pode conduzir a um resultado surpreendente à primeira vista e como a elucidação deste resultado surpreendente pode ajudar na sedimentação dos conceitos de Mecânica Quântica envolvidos. O problema é o de uma partícula no interior de um poço quadrado infinito, o qual tem sua largura repentinamente dobrada. A surpresa está em que resultados diferentes podem ser obtidos quando o poço é tratado como não simétrico ou como simétrico, embora, como se sabe bem, ambos sejam fisicamente idênticos.
\end{abstract}

Palavras-chave: Poço quadrado infinito, poço quadrado dobrado, perturbação repentina, autofunções.

In this work, we would like to point out how an usually recommended exercise in Quantum Mechanics textbooks can lead to a surprising result at first, and how the clarification of this surprising result can help to reinforce ideas of Quantum Mechanics. We consider the problem of a particle in an infinite square well which suddenly has its width doubled. The surprise arrives in the fact that different results can be obtained whether the well is considered non-symmetrical or symmetrical, even though both are physically identical, as it is well-known.

Keywords: Infinite square well; Quantum Mechanics, sudden approximation, Solutions of wave equations, bound states.

\section{Introdução}

Em geral, todo aluno na disciplina de Mecânica Quântica da graduação aprende que um poço quadrado, infinito, unidimensional e não simétrico é fisicamente idêntico a um simétrico de mesma largura, nada de diferente havendo, exceto por uma inofensiva translação da origem do sistema de coordenadas. As funções de onda, o espectro de energia e as probabilidades não são afetadas pela abordagem escolhida.

Assim, em um problema no qual uma partícula de massa $m$ que se encontra no estado fundamental de um poço quadrado infinito quando sua largura é repentinamente dobrada, torna-se surpreendente e difícil para os alunos explicarem em uma abordagem quântica porque são obtidos resultados diferentes para as probabilidades da partícula ser encontrada nos novos estados de energia do poço de largura dobrada dependendo de como o poço é tratado, se na abordagem não simétrica ou na simétrica.

Este problema é de simples resolução no que é solicitado, em geral o cálculo da probabilidade de encontrar a partícula no estado fundamental do novo poço de largura

*Endereço de correspondência: jose.nogueira@ufes.br dobrada. Entretanto, pode se tornar muito interessante e uma fonte rica para se discutir e sedimentar conceitos da Mecânica Quântica. Para tanto, conforme veremos, necessita de um enunciado mais cuidadoso, a fim de não introduzir ambiguidades em sua interpretação.

O objetivo deste trabalho é, então, chamar a atenção para o problema e discutir as questões relevantes envolvidas, visto que, até onde sabemos, tais discussões não são encontradas nos livros-texto de Mecânica Quântica.

É importante salientarmos que, embora a literatura sobre o poço quadrado infinito seja muito extensa, não encontramos nada que trate do resultado surpreendente discutido neste trabalho.

O trabalho está organizado como segue: na Seção 2 . apresentamos o problema e sua solução considerando o poço não simétrico; na Seção 3 mostramos que, ao considerar o poço simétrico, a solução fica diferente da obtida anteriormente; nas Seções 4 e 5 . discutimos a elucidação deste resultado surpreendente; nas Seções 6 e 7 discutimos outros resultados importantes, tais como os relativos a energias permitidas e sobre a evolução temporal do sistema. Na Seção 8, apresentamos nossas conclusões e fazemos um breve relato sobre os trabalhos que temos encontrado na literatura sobre o potencial 
de um poço quadrado infinito. Optamos por fazê-lo nas Considerações Finais e não na Introdução a fim de que não seja perdido o foco no objetivo deste trabalho. Ainda, também com a intenção de não desviar a atenção do leitor dos aspectos relevantes que aqui se quer tratar, foram anexados dois Apêndices nos quais podem ser encontrados os cálculos das probabilidades apresentadas no texto para o caso do poço não simétrico, Apêndice A, e para o caso simétrico, Apêndice B. Há também um Apêndice $\mathrm{C}$, onde é apresentado um relato mais extenso do material encontrado na literatura sobre o potencial de um poço quadrado infinito.

\section{O Problema}

O problema é um exercício que pode ser encontrado em livros-texto de Mecânica Quântica dos cursos de graduação em Física [1] e sua solução é relativamente simples:

Considere que uma partícula de massa $m$, por exemplo um elétron, encontra-se no estado fundamental de um poço quadrado infinito 1-dimensional de largura a. No instante $t=0$, repentinamente, a largura do poço é dobrada, isto é, passa a ser $2 a$. Qual a probabilidade de encontrar a partícula no estado fundamental do novo poço?

A fim de encontrarmos a probabilidade solicitada, vamos considerar que, inicialmente, as paredes do poço infinito estejam em $x=0$ e $x=a$, isto é, um poço não simétrico. Note que fisicamente seria indiferente se considerássemos as paredes em $x=-\frac{a}{2}$ e $x=\frac{a}{2}$, isto é, um poço simétrico. Então, para $t<0$, como é bem conhecido, o vetor de estado da partícula na representação do espaço de posições (autofunção de onda do estado fundamental) é

$$
\left\langle x \mid \varphi_{1}\right\rangle=\varphi_{1}(x)= \begin{cases}\sqrt{2 / a} \operatorname{sen}(\pi x / a), & 0 \leq x \leq a \\ 0, & x \leq 0 \text { ou } x \geq a .\end{cases}
$$

Agora, se a hamiltoniana de um sistema é alterada repentinamente, o vetor de estado do sistema não deve praticamente ser modificado $1-5]$. Então, a probabilidade da partícula ser encontrada no estado fundamental do novo poço é dada por (veja Apêndice A)

$$
\mathbb{P}_{1}=\left|\left\langle\phi_{1} \mid \varphi_{1}\right\rangle\right|^{2}
$$

onde $\left|\phi_{1}\right\rangle$ é o vetor de estado fundamental do novo poço. Considerando que a parede em $x=a$ é movida para $x=$ $2 a$, na representação do espaço de posições, a autofunção de onda do estado fundamental do novo poço é dada por
Assim, das Eqs. (1) e (3) temos

$$
\left\langle\phi_{1} \mid \varphi_{1}\right\rangle=\frac{\sqrt{2}}{a} \int_{0}^{a} d x \operatorname{sen}(\pi x / 2 a) \operatorname{sen}(\pi x / a)=\frac{4 \sqrt{2}}{3 \pi} .
$$

Note que o limite superior da integral é $a$ e não $2 a$, pois $\varphi_{1}(x)$ é diferente de zero apenas no intervalo $(0, a)$. Fisicamente, podemos interpretar isso como se a partícula não tivesse tido tempo para ocupar a nova região livre no interior do poçd ${ }^{1}$ Portanto, a probabilidade de encontrarmos a partícula no estado fundamental do novo poço é

$$
\mathbb{P}_{1}=\frac{32}{9 \pi^{2}} \cong 36 \%
$$

\section{O Resultado Surpreendente}

Agora, vamos considerar que, inicialmente, as paredes do poço infinito estejam em $x=-\frac{a}{2}$ e $x=\frac{a}{2}$, isto é, um poço simétrico. Então, para $t<0$, como também é bem conhecido, o vetor de estado da partícula na representação do espaço de posições ${ }^{2}$ é

$$
\begin{aligned}
& \left\langle x \mid \varphi_{1}^{s}\right\rangle=\varphi_{1}^{s}(x)= \\
& \begin{cases}\sqrt{2 / a} \cos (\pi x / a), & -\frac{a}{2} \leq x \leq \frac{a}{2} \\
0, & x \leq-\frac{a}{2} \text { ou } x \geq \frac{a}{2}\end{cases}
\end{aligned}
$$

Neste caso, a probabilidade da partícula ser encontrada no estado fundamental do novo poço é dada por (veja Apêndice B)

$$
\mathbb{P}_{1}^{s}=\left|\left\langle\phi_{1}^{s} \mid \varphi_{1}^{s}\right\rangle\right|^{2},
$$

onde $\left|\phi_{1}^{s}\right\rangle$ é o vetor de estado fundamental do novo poço simétrico, o qual é dado, na representação do espaço de posições, por

$$
\begin{aligned}
& \left\langle x \mid \phi_{1}^{s}\right\rangle=\phi_{1}^{s}(x)= \\
& \begin{cases}\sqrt{1 / a} \cos (\pi x / 2 a), & -a \leq x \leq a \\
0, & x \leq-a \text { ou } x \geq a .\end{cases}
\end{aligned}
$$

Assim, das Eqs. (6) e (8) temos

$$
\left\langle\phi_{1}^{s} \mid \varphi_{1}^{s}\right\rangle=\frac{\sqrt{2}}{a} \int_{-\frac{a}{2}}^{\frac{a}{2}} d x \cos (\pi x / 2 a) \cos (\pi x / a)=\frac{8}{3 \pi} .
$$

Portanto, a probabilidade de encontrarmos a partícula no estado fundamental do novo poço simétrico é

$$
\mathbb{P}_{1}^{s}=\frac{64}{9 \pi^{2}} \cong 72 \%,
$$

que é diferente do resultado obtido na seção anterior. Mas isso é o contrário do que, aparentemente, poderíamos esperar, pois, em ambos os casos, temos inicialmente

${ }^{1}$ Como veremos na Seção 7 a probabilidade da partícula ser encontrada em $a \leq x<2 a$ pode não ser nula conforme o sistema evolui no tempo. Na verdade, oscila periodicamente entre 0 e $100 \%$.

${ }_{2}$ Nós usamos o superíndice $s$ para indicar os estados do poço simétrico. 
uma partícula de massa $m$ no estado fundamental de um poço quadrado infinito, de largura $a$, e calculamos a probabilidade da partícula ser encontrada no estado fundamental do poço agora de largura $2 a$. A física do problema não deve depender se consideramos matematicamente o poço simétrico ou não simétrico. Então, como explicar os diferentes resultados obtidos?

\section{A Elucidação do Resultado Surpreendente}

É claro que poços infinitos simétrico e não simétrico de larguras iguais são fisicamente os mesmos, e que o estado fundamental também é o mesmo ${ }^{3}$. Então, por que obtemos diferentes resultados? A resposta está em que no poço não simétrico realizamos um deslocamento apenas da parede direita do poço de uma distância $a$, enquanto no caso do poço simétrico deslocamos ambas as paredes de uma distância $\frac{a}{2}$. Assim, embora o sistema final seja idêntico em ambos os casos (isto é, um poço infinito de largura $2 a$ ) e seus estados iniciais também pareçam ser os mesmos (isto é, o estado fundamental do poço de largura a), isso não é verdade, pois em relação à partícula, os processos pelos quais o poço tem sua largura dobrada são distinto\$ 4 o que faz com que os estados iniciais dos poços de larguras duplicadas não sejam os mesmos em ambos os casos.

A Fig. 1 mostra claramente o que estamos afirmando. Como podemos ver, a função de onda da partícula ocupa a mesma região dentro do poço de largura $a$ não simétrico, Fig. 1a, bem como no simétrico, Fig. 1b, antes de a largura ser dobrada. Entretanto, o mesmo não é verdade quando os poços têm largura $2 a$. Enquanto no poço não simétrico a função de onda inicial da partícula ocupa apenas a metade esquerda do poço, no poço simétrico ela está simetricamente distribuída em relação ao eixo que passa pelo centro do poço.

Matematematicamente, é fácil ver que os estados iniciais dos poços dobrados não simétrico e simétrico não são os mesmos, pois, enquanto as funções de onda dos estados estacionários (autofunções de energia) do poço dobrado $\phi_{n}(x)$ são levadas em $\phi_{n}^{s}(x)$ pela translação $x \rightarrow x-a$, a função de onda $\varphi_{1}^{s}(x)$ não pode ser obtida de $\varphi_{1}(x)$

${ }^{3}$ Note que os estados são não degenerados e diferentes são as representações matemática para um mesmo estado. Um poço não simétrico sempre pode ser tornado simétrico, ou vice-versa, por uma simples translação da origem. Seja $x$ a coordenada para um poço infinito não simétrico de largura $a$, e façamos uma translação da origem tal que $x \rightarrow x-\frac{a}{2}$. Então, as autofunções do poço não simétrico,

$$
\begin{aligned}
& \sqrt{\frac{2}{a}} \operatorname{sen}\left(\frac{n \pi x}{a}\right) \rightarrow \sqrt{\frac{2}{a}} \operatorname{sen}\left(\frac{n \pi x}{a}-\frac{n \pi}{2}\right) \rightarrow \\
& \begin{cases}\sqrt{2 / a} \cos (n \pi x / a), & n=1,3,5, \ldots \\
\sqrt{2 / a} \operatorname{sen}(n \pi x / a), & n=2,4,6, \ldots,\end{cases}
\end{aligned}
$$

se tornam aquelas de um poço simétrico de largura $a$.

${ }^{4}$ Note que as posições físicas ocupadas pelas paredes do poço não serão as mesmas, na comparação entre os dois casos.
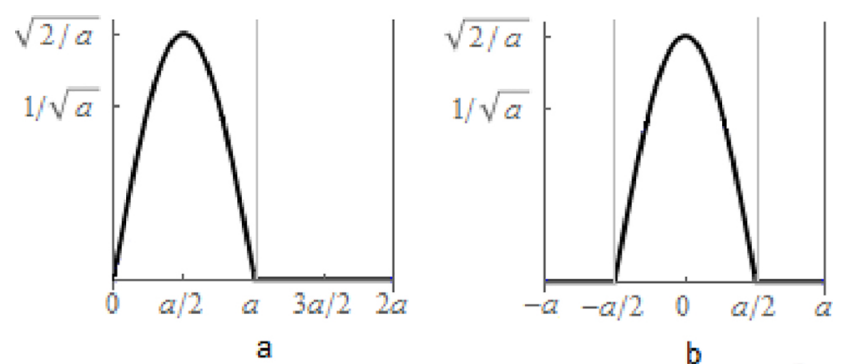

Figura 1: Partícula no estado fundamental de um poço infinito de largura $a$ que será o estado inicial de um poço de largura $2 a$ quando (a) no caso não simétrico, a parede da direita foi deslocada para a direita de uma distância $a$, e quando (b) no caso simétrico, a parede da esquerda foi deslocada para a esquerda de uma distância $\frac{a}{2}$ e a da direita foi deslocada para a direita também de $\frac{a}{2}$.

pela mesma translação. Essa translação levaria à função $\tilde{\varphi}_{1}^{s}(x)$, dada abaixo:

$$
\begin{aligned}
& \varphi_{1}^{s}(x) \neq \tilde{\varphi}_{1}^{s}(x)= \\
& \begin{cases}\sqrt{2 / a} \operatorname{sen}(\pi x / a), & -a \leq x \leq 0 \\
0, & x \leq-a \text { ou } x \geq 0 .\end{cases}
\end{aligned}
$$

Agora, sendo os vetores de estado iniciais da partícula distintos nos dois casos, eles têm projeções diferentes sobre os vetores de estado estacionários, ou sobre as autofunções de energia, do poço de largura $2 a$ (veja Apêndice A):

$$
|\phi(0)\rangle=\left|\varphi_{1}\right\rangle=\frac{\sqrt{2}}{2}\left|\phi_{2}\right\rangle+\sum_{n=1,3,5, \ldots}^{\infty} \frac{4 \sqrt{2}}{\pi} \frac{(-1)^{\frac{n+1}{2}}}{\left(n^{2}-4\right)}\left|\phi_{n}\right\rangle,
$$

$$
\begin{aligned}
& \varphi_{1}(x)=\frac{\sqrt{2}}{2} \frac{1}{\sqrt{a}} \operatorname{sen}\left(\frac{\pi x}{a}\right)+\frac{4 \sqrt{2}}{\pi} \sum_{n=1,3,5, \ldots}^{\infty} \frac{(-1)^{\frac{n+1}{2}}}{\left(n^{2}-4\right)} \\
& \times \frac{1}{\sqrt{a}} \operatorname{sen}\left(\frac{n \pi x}{2 a}\right), 0 \leq x \leq a,
\end{aligned}
$$

e (veja Apêndice B) também

$$
\left|\phi^{s}(0)\right\rangle=\left|\varphi_{1}^{s}\right\rangle=\frac{8}{\pi} \sum_{n=1,3,5, \ldots}^{\infty} \frac{(-1)^{\frac{n+1}{2}}}{\left(n^{2}-4\right)}\left|\phi_{n}^{s}\right\rangle,
$$

$$
\begin{aligned}
& \varphi_{1}^{s}(x)=\frac{8}{\pi} \sum_{n=1,3,5, \ldots}^{\infty} \frac{(-1)^{\frac{n+1}{2}}}{\left(n^{2}-4\right)} \frac{1}{\sqrt{a}} \cos \left(\frac{n \pi x}{2 a}\right), \\
& -\frac{a}{2} \leq x \leq \frac{a}{2}
\end{aligned}
$$

Portanto, são probabilidades distintas de encontrarmos a partícula em um determinado estado estacionário do 
poço de largura $2 a$. Na Fig. 2a estão representadas as funções de onda do estado inicial $\varphi_{1}(x)$ e do estado fundamental do poço não simétrico $\phi_{1}(x)$, enquanto na Fig. $2 \mathrm{~b}$ estão representadas as funções de onda do estado inicial $\varphi_{1}^{s}(x)$ e do estado fundamental $\phi_{1}^{s}(x)$ do poço simétrico de largura 2a. Nelas é possível ver que a intersecçã ${ }^{5}$ do estado inicial e do fundamental do poço não simétrico, Fig. 2a, é menor que o do simétrico, Fig. $2 \mathrm{~b}$.

\section{Processos Físicos Equivalentes}

Como afirmamos na seção anterior, as probabilidades resultaram diferentes porque os processos através dos quais a largura do poço quadrado infinito é dobrada são distintos. Então, devemos esperar que processos idênticos devam levar a resultados iguais para a probabilidade de encontrarmos a partícula no estado fundamental do novo poço de largura $2 a$.

Vamos considerar que a parede direita do poço simétrico seja levada para a direita de uma distância $a$, como no caso do poço não simétrico. O poço simétrico se torna agora um poço não simétrico, cujas paredes se encontram em $x=-\frac{a}{2}$ e $x=\frac{3 a}{2}$. A autofunção de onda do estado fundamental desse poço é dada por

$\tilde{\phi}_{1}(x)= \begin{cases}\sqrt{1 / a} \operatorname{sen}\left[\frac{\pi}{2 a}\left(x+\frac{a}{2}\right)\right], & -\frac{a}{2} \leq x \leq \frac{3 a}{2} \\ 0, & x \leq-\frac{a}{2} \text { ou } x \geq \frac{3 a}{2} .\end{cases}$

Então, a probabilidade de encontrarmos a partícula no estado fundamental desse poço será dada por

$$
\tilde{\mathbb{P}}_{1}=\left|\left\langle\tilde{\phi}_{1} \mid \varphi_{1}\right\rangle\right|^{2}=\frac{32}{9 \pi^{2}}=\mathbb{P}_{1} \cong 36 \%
$$

Como havíamos afirmado, obtém-se o mesmo resultado do caso não simétrico, uma vez que agora a função de onda inicial é a mesma para os dois casos.

\section{Uma Discussão sobre as Energias}

Em uma medida da energia da partícula no novo poço com paredes em $x=0$ e $x=2 a$, a probabilidade de

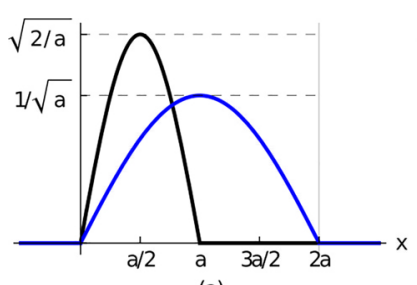

(a)

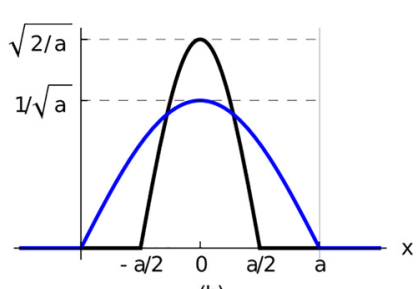

(b)
Figura 2: Representação das funções de onda do estado inicial e fundamental para os poços de largura $2 a$ não simétrico (a) e simétrico (b).

${ }^{5}$ Por intersecção aqui queremos dizer a região onde pelo menos uma delas não é nula. Note que onde uma delas é nula $\left(\varphi_{1}\right.$ ou $\left.\varphi_{1}^{s}\right)$ o produto $\varphi_{1}(x) \phi_{1}(x)$ ou $\varphi_{1}^{s}(x) \phi_{1}^{s}(x)$ se anula. obtermos o resultado $E_{n}^{\prime}=\frac{\hbar^{2} \pi^{2} n^{2}}{8 m a^{2}}$ é dada por (veja Apêndice A)

$$
\begin{aligned}
& \mathbb{P}_{n}=\left|\left\langle\phi_{n} \mid \varphi_{1}\right\rangle\right|^{2}=\left|c_{n}\right|^{2} \\
& = \begin{cases}\frac{32}{\pi^{2}\left(n^{2}-4\right)^{2}}, & n=1,3,5, \ldots \\
1 / 2, & n=2 \\
0, & n=4,6,8, \ldots\end{cases}
\end{aligned}
$$

Do resultado acima, podemos concluir que o valor mais provável de energia é $E_{2}^{\prime}=\frac{\pi^{2} \hbar^{2}}{2 m a^{2}}=E_{1}$, coincidentemente a energia inicial da partícula no poço original de largura $a$, com probabilidade de medida igual a $\mathbb{P}_{2}=\frac{1}{2}(50 \%)$. As demais probabilidades decrescem rapidamente com $n$, e as duas seguintes mais prováveis são $E_{1}^{\prime}=\frac{\pi^{2} \hbar^{2}}{8 m a^{2}}=\frac{E_{1}}{4}$ e $E_{3}^{\prime}=\frac{9 \pi^{2} \hbar^{2}}{8 m a^{2}}=\frac{9 E_{1}}{4}$ com respectivas probabilidades

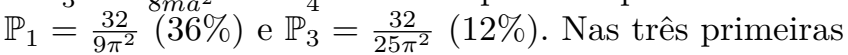
energias se concentram quase $99 \%$ das chances de medida!

Na Fig. 3 estão representadas a função de onda do estado fundamental $\left|\varphi_{1}\right\rangle$ do poço infinito de largura $a$ (linha contínua) e as funções de onda do estado fundamental $\left|\phi_{1}\right\rangle$ (linha pontilhada) e do primeiro estado excitado $\left|\phi_{2}\right\rangle$ (linha tracejada) do poço de largura $2 a$. Como podemos ver, a área de intersecção das curvas $\varphi_{1}(x)$ e $\phi_{2}(x)$ é maior que a área de intersecção das curvas $\varphi_{1}(x)$ e $\phi_{1}(x)$, resultando, então, em uma maior probabilidade de encontrar $E_{2}^{\prime}$ do que $E_{1}^{\prime}$ em uma medida da energia.

Já para o poço com parede em $x=-a$ e $x=a$ a probabilidade de, em uma medida da energia da partícula, encontrarmos $E_{n}^{s \prime}=\frac{\hbar^{2} \pi^{2} n^{2}}{8 m a^{2}}$ é dada por (veja Apêndice B)

$$
\begin{aligned}
& \mathbb{P}_{n}^{s}=\left|\left\langle\phi_{n}^{s} \mid \varphi_{1}^{s}\right\rangle\right|^{2}=\left|c_{n}^{s}\right|^{2} \\
& = \begin{cases}\frac{64}{\pi^{2}\left(n^{2}-4\right)^{2}}, & n=1,3,5, \ldots \\
0, & n=4,6,8, \ldots\end{cases}
\end{aligned}
$$

Comparativamente à abordagem não simétrica anterior do poço, há uma clara diferença no resultado obtido. O autovalor de energia $E_{2}^{s \prime}=E_{1}$ nem aparece no espectro provável de ser medido! Não há mais a probabilidade de encontrarmos, em uma medida da energia da partícula,

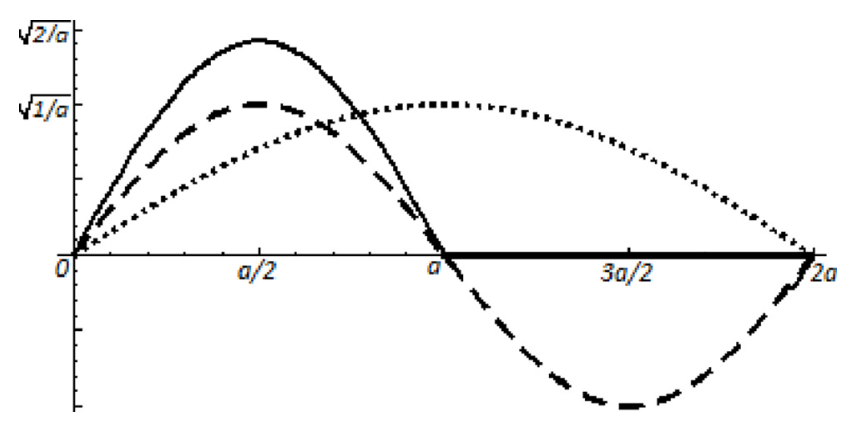

Figura 3: Função de onda do estado fundamental $\left|\varphi_{1}\right\rangle$ do poço infinito de largura $a$ (linha contínua) e funções de onda do estado fundamental $\left|\phi_{1}\right\rangle$ (linha pontilhada) e primeiro estado excitado $\left|\phi_{2}\right\rangle$ (linha tracejada) do poço não simétrico de largura $2 a$. 
o mesmo valor inicial da energia da partícula. A energia mais provável de ser encontrada agora é $E_{1}^{s \prime}=\frac{E_{1}}{4}$, com probabilidade de medida $\mathbb{P}_{1}^{s}=\frac{64}{9 \pi^{2}}(72 \%)$. As duas próximas energias mais prováveis de serem medidas são $E_{3}^{s \prime}=\frac{9 E_{1}}{4}$ e $E_{5}^{s \prime}=\frac{25 E_{1}}{4}$, de probabilidades $\mathbb{P}_{3}^{s}=\frac{64}{25 \pi^{2}}$ $(26 \%)$ e $\mathbb{P}_{5}^{s}=\frac{64}{441 \pi^{2}}(1,5 \%)$, respectivamente. É fácil ver na Fig. 4 que a área de intersecção da função de onda do estado fundamental $\left|\varphi_{1}^{s}\right\rangle$ (linha contínua) com $\left|\phi_{1}^{s}\right\rangle$ (linha pontilhada) é muito maior que com $\left|\phi_{3}^{s}\right\rangle$ (linha tracejada) ${ }^{6}$

Como é fácil ver, dentre as energias que podem ser medidas em cada caso, a mais provável de ser obtida é aquela que mais se aproxima da energia inicial da partícula. Um resultado que intuitivamente poderíamos esperar.

Uma vez que o deslocamento da(s) parede(s) do poço $\operatorname{ocorre}(\mathrm{m})$ repentinamente, a partícula não tem tempo para trocar energia com sua vizinhança. Assim, esperamos que o valor médio da energia da partícula em ambos os casos, poço não simétrico e simétrico de largura $2 a$, seja igual à energia inicial da partícula. O que pode ser facilmente comprovado (ver Equação (A12), no Apêndice A) pelo cálculo do valor médio em ambos os casos:

$$
\begin{aligned}
& \left\langle E^{\prime}\right\rangle=\sum_{n=0}^{\infty} \mathbb{P}_{n} E_{n}^{\prime}=\frac{1}{2} E_{1} \\
& +\frac{E_{1}}{4} \cdot \frac{32}{\pi^{2}} \sum_{n=1,3,5, \ldots} \frac{n^{2}}{\left(n^{2}-4\right)^{2}}=E_{1},
\end{aligned}
$$

e

$$
\left\langle E^{s \prime}\right\rangle=\sum_{n=0}^{\infty} \mathbb{P}_{n}^{s} E_{n}^{s \prime}=\frac{E_{1}}{4} \cdot \frac{64}{\pi^{2}} \sum_{n=1,3,5, \ldots} \frac{n^{2}}{\left(n^{2}-4\right)^{2}}=E_{1} .
$$

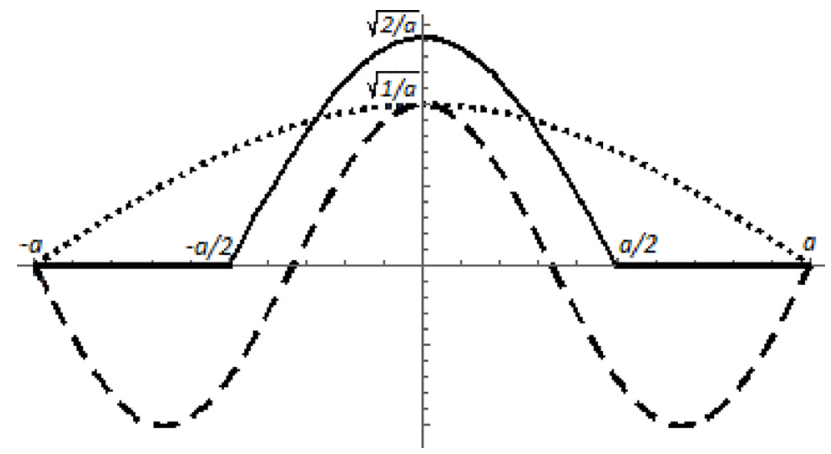

Figura 4: Função de onda do estado fundamental $\left|\varphi_{1}^{s}\right\rangle$ do poço infinito de largura $a$ (linha contínua) e funções de onda do estado fundamental $\left|\phi_{1}^{s}\right\rangle$ (linha pontilhada) e primeiro estado excitado $\left|\phi_{3}^{s}\right\rangle$ (linha tracejada) do poço simétrico de largura $2 a$.

\footnotetext{
${ }^{6}$ Note que nas regiões $\left(-\frac{a}{2},-\frac{a}{3}\right)$ e $\left(\frac{a}{3}, \frac{a}{2}\right)$ o produto $\phi_{3}^{s}(x) \varphi_{1}^{s}(x)$ é negativo.
}

\section{Evolução Temporal do Sistema}

Uma vez que o deslocamento da(s) parede(s) ocorre $(\mathrm{m})$ repentinamente, a probabilidade de encontrarmos a partícula na região entre $a$ e $2 a$ (entre $-a$ e $-\frac{a}{2}$ ou $\frac{a}{2}$ e $a$ ) imediatamente após o deslocamento da(s) parede(s) é nula, pois a partícula não teve tempo para ocupar essa(s) região(ões). Assim, esperamos que com a evolução temporal do sistema a partícula possa ser encontrada nessa(s) região(ões). A fim de provarmos essa nossa afirmação para o caso do poço infinito não simétrico, vamos calcular a probabilidade da partícula ser encontrada na região entre $a$ e $2 a$. Da Eq. (12) temos

$$
\begin{aligned}
& |\phi(t)\rangle=\frac{\sqrt{2}}{2} e^{-\frac{i}{\hbar} E_{2} t}\left|\phi_{2}\right\rangle \\
& +\sum_{n=1,3,5, \ldots}^{\infty} \frac{4 \sqrt{2}}{\pi} \frac{(-1)^{\frac{n+1}{2}}}{\left(n^{2}-4\right)} e^{-\frac{i}{\hbar} E_{n} t}\left|\phi_{n}\right\rangle .
\end{aligned}
$$

Então, em uma medida da posição da partícula no poço infinito de largura $2 a$, a probabilidade de que ela seja encontrada na região $a \leq x<2 a$, em um instante $t \geq 0$, é

$$
\begin{aligned}
& \mathbb{P}(x \geq a, t)=\int_{a}^{2 a}|\phi(x, t)|^{2} d x \\
& \mathbb{P}(x \geq a, t)=\frac{1}{2}-\frac{32}{\pi^{2}} \sum_{n=1,3,5, \ldots}^{\infty} \frac{1}{\left(n^{2}-4\right)^{2}} \\
& \times \cos \left[\frac{\pi^{2} \hbar\left(n^{2}-4\right) t}{8 m a^{2}}\right] .
\end{aligned}
$$

Note que:

i) $\mathbb{P}(x \geq a, t)=0$ quando $t=\frac{8 m a^{2}}{\pi \hbar} n_{p}$, com $n_{p}=$ $0,2,4, \ldots ;$

ii) $\mathbb{P}(x \geq a, t)=1(100 \%)$ quando $t=\frac{8 m a^{2}}{\pi \hbar} n_{i}$, com $n_{i}=1,3,5, \ldots$.

Agora, a probabilidade de encontrarmos a partícula na região $0<x<a$, em um instante $t \geq 0$, é

$$
\begin{aligned}
& \mathbb{P}(x<a, t)=\int_{0}^{a}|\phi(x, t)|^{2} d x=\frac{1}{2} \\
& +\frac{32}{\pi^{2}} \sum_{n=1,3,5, \ldots}^{\infty} \frac{1}{\left(n^{2}-4\right)^{2}} \cos \left[\frac{\pi^{2} \hbar\left(n^{2}-4\right) t}{8 m a^{2}}\right]
\end{aligned}
$$

Assim, a probabilidade de encontrarmos a partícula na região $a<x<2 a$ ou $0<x<a$ é periódica, com período igual a $\tau=2 \frac{8 m a^{2}}{\pi \hbar}$, como pode ser visto facilmente nas Figs. 5 e 6. Também, podemos notar que o gráfico da probabilidade $\mathbb{P}(x \geq a, t)$ na Fig. 5 está defasado do gráfico da probabilidade $\mathbb{P}(x<a, t)$ na Fig. 6 de meio período, portanto, quando a probabilidade de encontrarmos a partícula em $0<x<a$ é máxima, isto é, $100 \%$, em $a<x<2 a$ é mínima, isto é, zero. É claro que $\mathbb{P}(x<a, t)+\mathbb{P}(x \geq a, t)=1$, pois a partícula deve estar em algum ponto no interior do poço. 


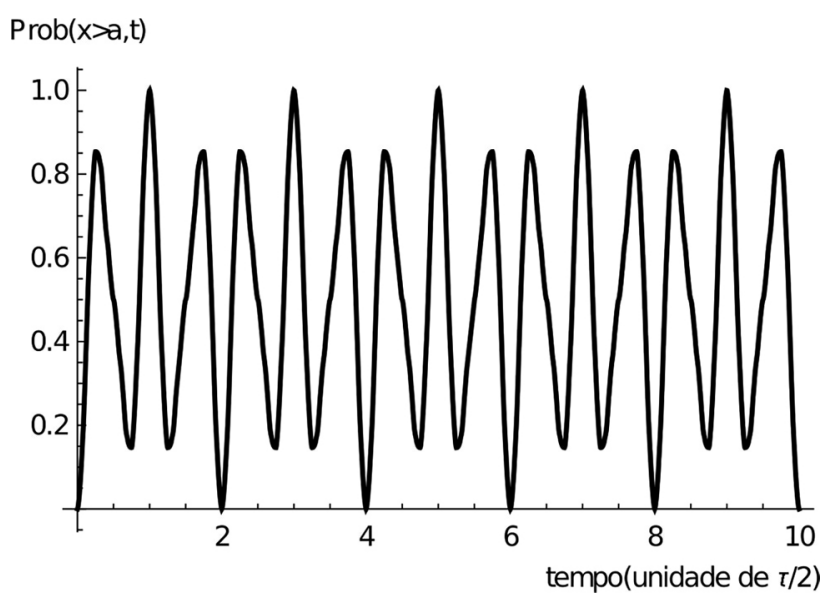

Figura 5: Probabilidade da partícula ser encontrada na região $a<x<2 a$ em função do tempo. A unidade de tempo é a metade do período, $\frac{\tau}{2}=\frac{8 m a^{2}}{\pi \hbar}$.

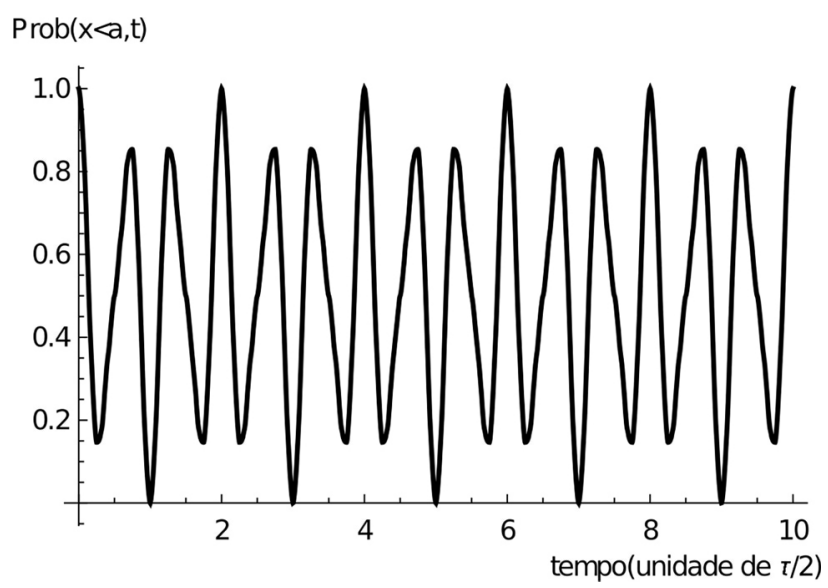

Figura 6: Probabilidade da partícula ser encontrada na região $0<x<a$ em função do tempo. A unidade de tempo é a metade do período, $\frac{\tau}{2}=\frac{8 m a^{2}}{\pi \hbar}$.

\section{Conclusões e Considerações Finais}

Neste trabalho, tratamos o problema de uma partícula de massa $m$ que encontra-se inicialmente no estado fundamental de um poço quadrado infinito cuja largura é repentinamente dobrada. Vimos que a probabilidade de encontrarmos a partícula no estado fundamental do novo poço de largura duplicada é diferente quando consideramos o poço como sendo não simétrico ou como simétrico. Então, alguma coisa parece estar fora do lugar aqui! Esse resultado poderia até nos confundir de imediato, pois parece ir contra o que seria esperado, visto que poços quadrados infinitos não simétricos e simétricos de iguais distâncias entre as paredes são fisicamente idênticos e a condição inicial é a mesma, a saber, o estado fundamental do poço de largura não dobrada. Assim, aqui existe um resultado surpreendente à primeira vista.

Entretanto, uma observação mais cuidadosa mostra que, na verdade, os dois casos não são fisicamente idênticos, pois a forma como o poço tem sua largura duplicada é diferente em cada caso, isto é, o(s) deslocamento da(s) parede(s) ocorre $(\mathrm{m})$ de modos diferentes. É claro que disso não dependem os níveis de energia do poço, pois, como é bem sabido, estes só dependem da largura do poço. Então, como esses diferentes deslocamentos das paredes estão relacionados com a descrição quântica do sistema? A resposta é que, na verdade, envolvem diferentes condições iniciais. Isto é, estando a partícula em determinado estado do poço não dobrado, sua distribuição de probabilidade em relação às paredes do poço está bem definida (veja Fig. 1), então diferentes deslocamentos das paredes fazem com que a probabilidade inicial se distribua de formas diferentes no interior do poço, o que significa dizer que as funções de onda iniciais não serão as mesmas. Assim, é através das funções de onda iniciais, as quais serão diferentes para diferentes deslocamentos das paredes, que do ponto de vista quântico são descritos os diferentes deslocamentos. Portanto, a relevância está no modo como as paredes são deslocadas e não na forma como o poço é tratado, se como simétrico ou não. Desta forma, a fim de evitar-se ambiguidades, o enunciado de problemas deste tipo deve deixar claro como o poço tem sua largura modificada [7], visto que as probabilidades de encontrarmos a partícula em certo estado do poço modificado depende crucialmente dessa ação.

Também vimos que o nível de energia mais provável da partícula ser encontrada imediatamente após o deslocamento da(s) parede(s) do poço é aquele cujo valor da energia esteja mais próximo do valor de sua energia inicial, no caso aqui discutido a energia do estado fundamental do poço não modificado.

Por fim, vimos que, imediatamente após o deslocamento da(s) parede(s) do poço, é nula a probabilidade de encontrarmos a partícula na nova região adicionada a seu interior, mas que pode ser diferente de zero com a evolução temporal do sistema. Tal resultado está de acordo ao esperado, pois sendo subitamente deslocada(s) a(s) parede(s) a partícula não ocupará a nova região disponível instantaneamente, mas com o passar do tempo ela poderá ser encontrada na nova região.

Trabalhos envolvendo o potencial de um poço quadrado infinito são largamente encontrados na literatura 7 devido à facilidade de sua resolução e ao fato de que muitos problemas físicos podem ser descritos aproximadamente pelo potencial de um poço quadrado infinito 8. Contudo, apesar de sua aparente simplicidade, o poço quadrado infinito esconde alguns problemas. O mais importante deles está relacionado à descontinuidade da derivada primeira de suas soluções (autofunções de energia) nas paredes do poço. Isso faz com que cálculos envolvendo o operador momento linear na representação de posição, $\langle x|\hat{p}| \varphi\rangle=-i \hbar \frac{\partial \varphi(x)}{\partial x}$, devam ser feitos com muito cuidado, isto é, usando um procedimento de limite, ou sejam realizados no espaço dos momentos, ou considerando

${ }^{7}$ De acordo com R. W. Robinett [8], é provável que o potencial de um poço quadrado infinito tenha aparecido pela primeira vez em 1930 no livro de N. F. Mott: An Outline of Wave Mechanics 17. 
um potencial suave. Isso, porém, não afeta os cálculos dos níveis de energia e das probabilidades da partícula no interior do poço ser encontrada em cada nível de energia 8 16]. Um dos efeitos da descontinuidade da derivada primeira da função de onda, $\frac{\partial \varphi(x)}{\partial x}$, causada pela altura infinita da parede do poço, é o desacordo das previsões para as propriedades da distribuição do momento linear realizadas nas representações do espaço de coordenadas e do espaço de momentos $8,9,11,12$. Em verdade, existe certa ambiguidade em se determinar as condições de contorno que devem ser satisfeitas pelas soluções, isto é, pelas funções de onda da partícula no interior do poço 2, 6, 8, 12, 18,22. Já em 1938, V. Rojansky sugeriu, em seu livro [6], que o problema das condições de contorno do poço quadrado infinito podia ser resolvido considerando o limite de um poço quadrado finito 8, 18. G. Bonneau, J. Faraut e G. Valent, com base nas possíveis extensões autoadjuntas do operador momento linear, concluiram que um poço quadrado de potencial infinito pode ser descrito como o limite de um finito, enquanto um potencial degrau infinito não pode ser considerado como o limite de um potencial degrau finito 23 .

Outro problema que surge no poço infinito é a aparente violação, ou não, do Teorema de Enhrenfest. Uma vez que os únicos lugares onde atuam forças sobre a partícula ocorrem nas paredes, onde a probabilidade de ela ser encontrada é nula, então poderíamos concluir que o valor médio da força sobre a partícula em qualquer estado seria nulo, portanto, violando o Teorema de Enhrenfest. D. S. Rokhsar contornou o problema também por tomar o limite do poço finito, e, assim salvando o Teorema de Enhrenfest 24]. Contudo, V. Alonso, S. De Vincenzo e A. González-Díaz apontaram que o teorema de Enhrenfest não é válido para qualquer das extensões autoadjuntas do operador hamiltoniano do poço infinito e mostraram que na forma usual ele não é aplicável ao poço quadrado infinito 25.

Com a intenção de discutir o problema da não localidade (ação a distância) 26 27 na mecânica quântica, L. Hardy 28] considerou uma partícula no interior de uma caixa, descrita pelo potencial de um poço quadrado infinito, que é dividida em duas partes iguais e essas partes são levadas uma para longe da outra. Entretanto, J. Gea-Banacloche argumentou que, na realidade, é praticamente impossível dividir a caixa em duas partes idênticas. Ele mostrou que, se uma parede divisória é introduzida muito lentamente (aproximação adiabática), a partícula sempre será encontrada no interior da parte maior após a divisória ser introduzida completamente, inviabilizando assim a discussão da não localidade 29 31]. V. B. S $\phi$ rdal e J. Bergli [32] contornaram o problema da assimetria mostrando que uma introdução assimétrica e não adiabática da parede divisória pode conduzir a probabilidades iguais $(50 \%)$ de encontrar a partícula em uma das partes. S. J. van Enk 33 considerou o caso de duas caixas idênticas, com uma partícula no estado fundamental em cada caixa. Ele mostrou que, após a divisão em partes iguais, separação e união de uma parte de cada caixa para formar novamente duas caixas, ambas as partículas terminam na mesma caixa, uma no estado fundamental e outra no primeiro estado excitado se forem férmions, ou ambas no estado fundamental ou no primeiro estado excitado se forem bósons. D. M. Greenberg mostrou que outro efeito de não localidade, passível de verificação experimental, seria o de uma diferença de fase na função de onda de uma partícula, inicialmente confinada em uma pequena região no interior de um poço quadrado infinito, causada pelo movimento de uma das paredes do poço 34].

O problema de um poço quadrado infinito com paredes móveis foi tratado por diversos autores [34 40]. A validade da aproximação adiabática foi mostrada para os casos de expansão e de contração lentas do poço e da aproximação repentina para uma rápida expansão do poço, entretanto, no caso de uma contração rápida do poço a aproximação repentina não é válida. Além disso, efeitos não triviais surgem devido ao movimento das paredes do poço, tais como divergências na incerteza e no valor médio da energia 36 38 e de não unitaridade (não normalização) na contração [35, 38, 39].

As simetrias exibidas em um poço quadrado infinito foram discutidas em 41,43$]$.

Devido a sua simplicidade o potencial de um poço quadrado infinito tem sido usado para exemplificar a aplicação do método variacional [44,45], do método do operador evolução 46], entre outros [36 47].

Uma discussão mais extensa dos trabalhos encontrados na literatura, acima citados, sobre o potencial de um poço infinito pode ser vista no Apêndice $\mathrm{C}$ pelos leitores interessados.

\section{Material suplementar}

O seguinte material suplementar está disponível online: Apêndice A: Poço quadrado infinito não simétrico Apêndice B: Poço quadrado infinito simétrico

Apêndice C: Breve discussão dos trabalhos encontrados na literatura sobre o potencial de um poço quadrado infinito

\section{Referências}

[1] N. Zettili, Quantum Mechanics - Concepts and Applications (Wiley, Nova York, 2009), $2^{\text {a }}$ ed.

[2] D.J. Ghiffiths, Introduction to Quantum Mechanics (Pearson Education, New Jersey, 2005), $2^{\mathrm{a}}$ ed.

[3] A. Messiah, Quantum Mechanics (North-Holland Publishing, Amsterdan, 1965), v. 2.

[4] R. Shankar, Principles of Quantum Mechanics (Plenum Press, New York, 1994), $2^{\mathrm{a}}$ ed.

[5] C. Cohen-Tannoudji, B. Dui e F. Laloe, Quantum Mechanics (Wiley, Paris, 1977), v. 1.

[6] V. Rojansky, Introductory Quantum Mechanics (PrinticeHall, New York, 1938), v. 1. 
[7] J.S. Townsend, A Fundamental Approach to Modern Physics (University Science Books, Mill Valley, 2010).

[8] R.W. Robinett, Phys. Report. 540, 25 (2014).

[9] F.L. Markley, Am. J. Phys. 40, 1545 (1972).

[10] A. Johannin-Gilles, R. Abjean, Y. Guern e M. Leriche, Am. J. Phys. 42, 702 (1974).

[11] F.E. Cummings, Am. J. Phys. 45, 158 (1977).

[12] G.M. Muha, J. Chem. Educ. 61, 414 (1984).

[13] R.W. Robinett, Am. J. Phys. 63, 823 (1995).

[14] M.A. Doncheski e R.W. Robinett, Eur. J. Phys. 21, 217 (2000).

[15] Z. Ahmed, S. kumar, D. Ghosh e J.A. Nathan, Eur. J. Phys. 39, e055402 (2018).

[16] Z. Ahmed, D. Ghosh, S. Kumar e J.A. Nathan, arXiv:1801.04730v2 (2018).

[17] N.F. Mott, An Outline of Wave Mechanics (Cambridge University Press, Cambridge, 1930).

[18] R. Seki, Am. J. Phys. 39, 929 (1971).

[19] D. Branson, Am. J. Phys. 47, 1000 (1979).

[20] M. Andrews, Am. J. Phys. 49, 281 (1981).

[21] D. Home e S. Sengupta, Am. J. Phys. 50, 552 (1982).

[22] D.H. McIntyre, C.A. Manogue e J. Tate, Quantum Mechanics (Pearson Education, New Jersey, 2012).

[23] G. Bonneau, J. Faraut e G. Valent, Am. J. Phys. 69, 322 (2001).

[24] D.S. Rokhsar, Am. J. Phys. 64, 1416 (1996).

[25] V. Alonso, S. De Vincenzo e A. González-Díaz, Il Nuovo Cimento 115B, 307 (2000).

[26] T. Norsen, Am. J. Phys. 73, 164 (2005).

[27] A. Shimony, Am. J. Phys. 73, 177 (2005).

[28] L. Hardy, Contemp. Phys. 39, 419 (1998).

[29] J. Gea-Banacloche, Am. J. Phys. 70, 307 (2002).

[30] M. Lakner e J. Peternej, Am. J. Phys. 71, 519 (2003).

[31] S. Ki Baek, S.D. Yi e M. Kim, Phys. Rev. A 94, 052124 (2016).

[32] V.B. Sørdal e J. Bergli, Phys. Rev. A 99, 022121 (2019).

[33] S.J. van Enk, Am. J. Phys. 77, 140 (2009).

[34] D.M. Greenberger, Physica B 151, 374 (1988).

[35] S.W. Doescher e M.H. Rice, Am. J. Phys. 37, 1246 (1969).

[36] D.W. Schlitt e C. Stutz, Am. J. Phys. 38, 70 (1970).

[37] A. Munier, J.R. Burgan, M. Feix e E. Fijalkow, J. Math. Phys. 22, 1219 (1981).

[38] D.N. Pinder, Am. J. Phys. 58, 54 (1990).

[39] V.V. Dodonov, A.B. Klimov e D.E. Nikonov, J. Math. Phys. 34, 3391 (1993).

[40] M.V. Berry e G. Klein, J. Phys. A: Math. Gen. 17, 1805 (1984).

[41] D. Aebersold e A. Langerman, Am. J. Phys. 45, 272 (1977).

[42] S.H. Dong e Z.Q. Ma, Am. J. Phys. 70, 520 (2002).

[43] A. Gangopadhyaya e J.V. Mallow, Am. J. Phys. 71, 182 (2003).

[44] V. Luaña, A. Otero-de la Roza, M.A. Blanco e J.M. Recio, Eur. J. Phys. 31, 101 (2010).

[45] M. Ögrenl e M. Carlsson, Eur. J. Phys. 32, L3 (2011).

[46] L.M.A. Aguilar, F.V. Luna, C. Robledo-Sánchez e M.L. Arroyo-Carrasco, Eur. J. Phys. 35, 025001 (2014).

[47] E. Peacock-López, Chem. Educator 16, 168 (2011). 